\title{
Radiation-Induced Pelvic Colorectal Obstruction After Neoadjuvant Chemoradiotherapy and Sphincter-Saving Surgery for Rectal Cancer: Is IMRT Better?
}

\section{Shenghui Huang}

Fujian Medical University Union Hospital

Minghong Chen

Fujian Medical University Union Hospital

\section{Xiaojie Wang}

Fujian Medical University Union Hospital

\section{Yuangui Chen}

Fujian Medical University Union Hospital

\section{Xingrong Lu}

Fujian Medical University Union Hospital

\section{Weizhong Jiang}

Fujian Medical University Union Hospital

\section{Ying Huang}

Fujian Medical University Union Hospital

\section{Pan Chi ( $\nabla$ Chipan620@hotmail.com )}

Fujian Medical University Union Hospital https://orcid.org/0000-0002-0004-1174

\section{Research}

Keywords: Radiation-induced fibrosis, Anastomotic stricture, Chemoradiotherapy, Rectal cancer

Posted Date: May 19th, 2020

DOl: https://doi.org/10.21203/rs.3.rs-28809/v1

License: (c) (1) This work is licensed under a Creative Commons Attribution 4.0 International License. Read Full License 


\section{Abstract}

\section{Purpose}

Radiation-induced pelvic colorectal obstruction (RIPCO) is a severe complication after neoadjuvant chemoradiotherapy (NCRT) and sphincter-saving surgery (SPS) with for rectal cancer. The incidence and risk factors remain unclear. This study aimed to evaluate the influences of anatomical and clinical parameters including radiation modalities on this complication.

\section{Methods}

From 2010 to 2018, data from patients who received NCRT and SPS with a diverting stoma for rectal cancer were collected. Patients with clinical parameters and complete pelvimetric parameters associated with RIPCO were included for univariate and multivariate analyses. Receiver operating characteristic curves were used to calculate the best cutoff value.

\section{Results}

A total of 726 patients with rectal carcinoma received NCRT and SPS with diverting stoma, and 157 patients had complete pelvimetric data. Eighteen of the 726 (2.5\%) patients developed RIPCO. Eleven patients only received recreation of a diverting stoma, and four were cured by intersphincteric resection with reconstruction of anastomosis and diverting stoma. In multivariate analysis, $\mathrm{BMI}(P=0.001)$, sacral depth $(P=0.046)$, and Intensity-modulated radiotherapy (IMRT) $(P<0.001)$ were independent predictors. The cutoff values of BMI and sacral depth were $21.1 \mathrm{~kg} / \mathrm{m}^{2}$ and $4.1 \mathrm{~cm}$, respectively. IMRT was independently associated with a lower incidence of RIPCO $(P<0.001)$. The four-factor scoring system showed that the sensitivity was $88.9 \%$, and the specificity was $85.6 \%$. The AUC was 0.921 .

\section{Conclusion}

RIPCO is a rare but severe complication after SPS following NCRT. A BMI more than $21.1 \mathrm{~kg} / \mathrm{m}^{2}$, a sacral depth less than $4.1 \mathrm{~cm}$, and IMRT may decrease the risk of RIPCO.

\section{Introduction}

Neoadjuvant chemoradiotherapy (NCRT) followed by radical surgery is a standard treatment for locally advanced rectal cancer [1]. Radiation brings tumor downstaging, with a pathological complete response rate of $10-30 \%$, a high degree of sphincter preservation with negative surgical margins, and good local control for rectal cancer [2]. However, due to the radiation injuries of bowel, nerves, and other soft tissues, severe low anterior resection syndrome, anastomotic and leakage, and stenosis are more frequent in patients with radiation than in those without radiation after sphincter-saving surgery $[3,4]$. In addition, patients who developed late-stage complications, including chronic radiation proctitis, anastomotic leakage, rectovaginal fistula, or bowel stricture, usually require another surgery even with a permanent stoma [4-8]. Our previous study suggested that 3.0\% (14/468) of patients who underwent NCRT and 
sphincter-saving surgery developed radiation-induced pelvic colorectal obstruction (RIPCO) [6], which is severely detrimental to the patients' quality of life. Therefore, Oncologists also recommended optimizing the indications of NCRT and the radiation modalities.

RIPCO is a rare but severe complication after neoadjuvant chemoradiotherapy (NCRT) and sphinctersaving surgery with diverting stoma. Few studies have demonstrated the incidence, clinical characteristics, and predictors of this complication [6]. A meta-analysis suggested that Intensitymodulated radiotherapy (IMRT) significantly reduced acute toxicity, including acute overall gastrointestinal toxicity more severe than grade 2 , diarrhea, and proctitis in locally advanced rectal cancer patients treated with NCRT compared to three-dimension conformal radiation (3DCRT) [9]. However, whether IMRT could reduce the radiation-induced intestinal fibrosis and the risk of RIPCO remains unknown. This study aimed to investigate the influences of pelvimetric and clinical parameters, including IMRT, on the incidence of RIPCO after NCRT and sphincter-saving surgery.

\section{Methods}

\section{Patient selection}

This is a single-center retrospective study. From September 2010 to October 2018, data from patients who received NCRT and sphincter-saving surgery with diverting stoma for middle and low rectal cancer were collected in this study. The data were collected from the colorectal cancer database after institutional approval. The exclusion criteria were as follows: (1) patients with synchronous cancer; (2) patients with obvious colonic ischemia; (3) patients who received extended colectomy, extended lymph node dissection, or multi-visceral resection; (4) patients who received postoperative radiotherapy. Patients with complete pelvimetric parameters were included for analysis of the clinical and pelvimetric parameters associated with RIPCO.

\section{Treatments}

Neoadjuvant radiotherapy included IMRT (45 Gy in 1.8-Gy fractions to the planning target volume (PTV) and 50 Gy in 2-Gy fractions to PTV boost as an integrated boost) or three-dimensionally conformal radiation (3DCRT) (The PTV was treated to $45 \mathrm{~Gy}$ in 1.8-Gy fractions followed by a 5.4-Gy boost to the PTV boost to a total dose of $50.4 \mathrm{~Gy}$ ). Concurrent chemotherapy was administered including two different regimes as follows: (1) 3-4 cycles of CAPOX regimens; (2) capecitabine $825 \mathrm{mg} / \mathrm{m} 2$ for 25 days, 7 days later followed by $1-2$ cycles of CAPOX regimens. Radical surgery was performed $8-10$ weeks after the end of the radiation. The patients received sphincter saving surgery 8-10 weeks after the end of the radiotherapy. The diverting stoma was closed 3 month after the primary surgery or 1 month after the end of adjuvant chemotherapy.

\section{Pelvic dimensions}

The 3.0-T high-resolution magnetic resonance imaging (MRI) and the Picture Archiving and Communication Systems (version 3.6, YLZ information Technology Co. Ltd.) were used for pelvic imaging 
and measurement, respectively. The radiologist was blinded to the clinical data and assessed the preoperative pelvic dimensions and the area before the primary surgery.

The definitions of pelvic parameters measured from MRI before the primary surgery have been described previously, including the pelvic inlet length, the pubic tubercle height, the pelvic outlet length, the sacral length, the sacral depth, the interspinous distance, the mesorectal area and the rectal area $[10,11]$.

\section{Definition}

RIPCO was defined as pelvic colorectal obstruction after NCRT and sphincter-saving surgery with a diagnosis of radiation-induced intestinal fibrosis by endoscopic or pathological examination (Fig. 1A-C). The colonic stricture was defined as a colonic lumen narrower than 1 fingerbreadth by digital rectal examination or an inner diameter less than $1.2 \mathrm{~cm}[6,12]$. It was quite different from a simple stricture of the anastomosis. The length of the colonic stricture above the anastomosis was equal to or more than $3 \mathrm{~cm}$. CT or MRI showed megacolon, a beak sign, and thickening wall of the stricture colon without any signs of pelvic recurrence (Fig. 1D-F). Before stoma closure, all patients underwent a digital rectal examination and water-soluble contrast enema using $76 \%$ Urografin to confirm the radiological healing of the anastomosis (Fig. 1G).

\section{Statistical Analysis}

We utilized the software IBM SPSS statistics version 25.0 (IBM Corp. Armonk, NY, USA) for statistical analyses. Univariate and multivariate analyses were used to identify the predictors associated with RIPCO. Significant preoperative variables in univariate analyses were entered into the multivariate Logistic analysis with a stepwise method. Receiver operating characteristic (ROC) analysis was used to evaluate the risk factors of RIPCO. The best cutoff value was calculated according to the Youden's index. The statistical significance was defined as a p-value less than 0.05 .

\section{Results}

\section{Patient characteristics}

A total of 726 patients received neoadjuvant chemoradiotherapy and sphincter-saving surgery with diverting stoma in the study period, and the data from 157 patients with complete pelvimetry data were included for analysis (Table 1). 
Table 1

Patient characteristics

\begin{tabular}{|c|c|c|c|c|c|}
\hline \multicolumn{2}{|l|}{ Variables } & $\begin{array}{l}\text { Total } \\
(n=157)\end{array}$ & $\begin{array}{l}\text { Without RIPCO } \\
(n=139)\end{array}$ & $\begin{array}{l}\text { With RIPCO } \\
(n=18)\end{array}$ & $P$-value \\
\hline \multirow[t]{2}{*}{ Sex } & male & 19 & 18 & 1 & 1.000 \\
\hline & female & 138 & 121 & 17 & \\
\hline \multicolumn{2}{|l|}{ Age,y } & $55 \pm 11$ & $56 \pm 11$ & $49 \pm 11$ & 0.007 \\
\hline \multicolumn{2}{|l|}{$\mathrm{BMI}\left(\mathrm{kg} / \mathrm{m}^{2}\right)$} & $22.9 \pm 2.9$ & $23.3 \pm 2.7$ & $20.3 \pm 2.7$ & $<0.001$ \\
\hline \multicolumn{2}{|c|}{ Distance to the anal verge, $\mathrm{cm}$} & $5.7 \pm 2.5$ & $5.8 \pm 2.6$ & $5.6 \pm 1.0$ & 0.533 \\
\hline \multirow[t]{5}{*}{ урT stage } & T0 & 35 & 29 & 6 & 0.001 \\
\hline & $\mathrm{T} 1$ & 19 & 18 & 1 & \\
\hline & $\mathrm{T} 2$ & 33 & 31 & 2 & \\
\hline & T3 & 68 & 61 & 7 & \\
\hline & $\mathrm{T} 4$ & 2 & 0 & 2 & \\
\hline \multirow[t]{3}{*}{ ypN } & NO & 118 & 105 & 13 & 0.573 \\
\hline & N1 & 30 & 27 & 3 & \\
\hline & N2 & 9 & 7 & 2 & \\
\hline \multirow[t]{2}{*}{ M } & MO & 142 & 125 & 17 & 1.000 \\
\hline & M1 & 15 & 14 & 1 & \\
\hline \multirow[t]{2}{*}{ Radiation modalities } & 3DCRT & 23 & 12 & 11 & $<0.001$ \\
\hline & IMRT & 134 & 127 & 7 & \\
\hline \multirow[t]{2}{*}{ Surgical procedure } & $\mathrm{ME}$ & 79 & 73 & 6 & 0.126 \\
\hline & ISR & 78 & 66 & 12 & \\
\hline \multicolumn{2}{|c|}{$\begin{array}{l}\text { Anastomotic leakage after stoma } \\
\text { closure }\end{array}$} & 11 & 8 & 3 & 0.116 \\
\hline \multicolumn{6}{|c|}{$\begin{array}{l}\text { RICOP, radiation-induced colonic stricture in pelvis; BMI, body mass index; ME, mesorectal excision; } \\
\text { ISR, intersphincteric resection }\end{array}$} \\
\hline
\end{tabular}

Eighteen patients (2.5\%) were diagnosed with RIPCO, and 17 patients were male. All 18 patients underwent sphincter-saving surgery with diverting stoma after NCRT. The median anastomosis was $3.5 \mathrm{~cm}$, with a range of $2-5 \mathrm{~cm}$ from the anal verge. The inner diameter of the stricture colon was $0.7 \pm$ $0.1 \mathrm{~cm}$. The proximal margins and distal margins were $12.5 \pm 2.6 \mathrm{~cm}$ and $2.0 \pm 0.8 \mathrm{~cm}$, respectively. The 
median length of the colonic stricture was $5.8 \mathrm{~cm}$, with a range of 3-10 $\mathrm{cm}$. We proposed that the colonic stricture above the anastomosis should be removed during primary sphincter-saving surgery. The proposed proximal margin was $19.0(14.8-25) \mathrm{cm}$. Three patients had minor anastomotic leakage (grade A) after the first stoma closure.

After developing RIPCO, patients received water-soluble contrast enema again and showed a beak sign with megacolon and colonic stricture in the pelvis (Fig. $1 \mathrm{H}$ ). The thickened colon in the pelvis could be detected by MRI or in the specimen (Fig. 1I).

\section{Univariate and multivariate analyses}

Univariate analysis showed that RIPCO was associated with age, body mass index (BMI), sacral depth, mesorectal area, IMRT, and ypT stage (Table 1 and Table 2). In multivariate analysis for significant preoperative predictors, $\mathrm{BMI}(P=0.001)$, sacral depth $(P=0.046)$, and IMRT $(P<0.001)$ were independently associated with RIPCO (Table 3).

Table 2

Anatomical parameters between patients with and without RIPCO

\begin{tabular}{|lllll|}
\hline Anatomical parameters & $\begin{array}{l}\text { Total } \\
(\mathrm{n}=157)\end{array}$ & Without RIPCO $(\mathrm{n}=139)$ & With RIPCO $(\mathrm{n}=18)$ & $P$-value \\
\hline Pelvic inlet length, $\mathrm{cm}$ & $11.3 \pm 1.2$ & $11.3 \pm 1.2$ & $11.4 \pm 1.0$ & 0.610 \\
\hline Pubic tubercle height, $\mathrm{cm}$ & $5.3 \pm 0.7$ & $5.3 \pm 0.8$ & $5.3 \pm 0.2$ & 0.931 \\
\hline Pelvic outlet length, cm & $7.8 \pm 0.8$ & $7.8 \pm 0.8$ & $7.7 \pm 0.9$ & 0.643 \\
\hline Sacral length, cm & $12.6 \pm 2.5$ & $12.6 \pm 2.7$ & $12.4 \pm 0.8$ & 0.726 \\
\hline Sacral depth, cm & $3.9 \pm 0.5$ & $3.8 \pm 0.5$ & $4.1 \pm 0.5$ & 0.028 \\
\hline Interspinous distance, cm & $8.8 \pm 1.0$ & $8.8 \pm 1.0$ & $8.9 \pm 1.0$ & 0.486 \\
\hline Mesorectal area, $\mathrm{cm}^{2}$ & $30.9 \pm 8.1$ & $31.3 \pm 8.4$ & $27.4 \pm 3.4$ & 0.001 \\
\hline Rectal area, $\mathrm{cm}^{2}$ & $9.3 \pm 4.7$ & $9.4 \pm 4.9$ & $9.1 \pm 3.4$ & 0.801 \\
\hline
\end{tabular}


Table 3

Multivariate analysis of preoperative parameters for RIPCO

\begin{tabular}{|lllll}
\hline Variables & $\mathrm{B}$ & $\mathrm{SE}$ & $\mathrm{P}$ & $\mathrm{OR}[95 \% \mathrm{Cl}]$ \\
\hline Age & -0.043 & 0.034 & 0.202 & $0.958[0.896-1.024]$ \\
\hline $\mathrm{BMI}\left(\mathrm{kg} / \mathrm{m}^{2}\right)$ & -0.608 & 0.190 & $\mathbf{0 . 0 0 1}$ & $0.544[0375-0.790]$ \\
\hline Sacral depth & 1.625 & 0.814 & $\mathbf{0 . 0 4 6}$ & $5.079[1.031-25.016]$ \\
\hline Mesorectal area & -0.071 & 0.058 & 0.220 & $0.931[0.831-1.044]$ \\
\hline IMRT & -2.893 & 0.803 & $<0.001$ & $0.055[0.011-0.267]$ \\
\hline Constant & 11.024 & 4.444 & 0.013 & - \\
\hline BMl, body mass index; IMRT, Intensity-modulated radiotherapy \\
\hline \multicolumn{5}{l}{} \\
\hline
\end{tabular}

\section{Receiver operating characteristic curves}

The ROC curves of BMI, sacral depth, and IMRT were obtained for predicting RIPCO. The cutoff values of $\mathrm{BMI}$ and sacral depth were $21.1 \mathrm{~kg} / \mathrm{m}^{2}$ and $4.1 \mathrm{~cm}$, respectively. The area under the curve of the threefactor system was 0.921 . With a cutoff value of 0.121 , the sensitivity was $88.9 \%$, and the specificity was $85.6 \%$ (Fig. 2).

\section{Treatments}

In the 18 patients with RIPCO, two patients with a stricture of $3 \mathrm{~cm}$ were cured by endoscopic dilation. Eleven patients $(61.1 \%)$ received recreation of the diverting stoma, and four patients $(22.2 \%)$ underwent intersphincteric resection with anastomosis resection, reconstruction, and diverting stoma. The narrow soft tissue of the pelvis was also enlarged by the incision of stricture and lysis of the adhesion. Radiationinduced colorectal fibrosis was diagnosed by pathological examination.

One of the 4 patients received endoscopic stent placement on day 19 after the first stoma closure and developed stent migration and a Clostridium difficile infection 5 days later (Fig. 3). We removed the stent and inserted a transanal tube. The Clostridium difficile infection was cured with vancomycin. One month later, he was cured by ISR with a diverting stoma. The stoma was closed 3 months later. One year after ISR, the Wexner score was 5.0.

\section{Discussion}

RIPCO is a rare but severe complication after neoadjuvant chemoradiotherapy and sphincter-saving surgery for rectal cancer. It is completely different from a simple anastomotic stricture. In fact, RIPCO is a chronic colonic obstruction in the pelvis due to radiation-induced fibrosis. The colonic stricture above the 
anastomosis can be as long as $10 \mathrm{~cm}$. This study suggested that BMI, sacral depth, and radiation modalities were associated with RIPCO.

According to the results observed in this study, the clinical manifestation of RIPCO was as follows. First, all patients received sphincter-saving surgery for middle and low rectal cancer following NCRT, and most patients were male. Second, most patients had symptoms and signs of left colonic obstruction after stoma closure, including abdominal distension, megacolon, and a bowel stricture of $3-10 \mathrm{~cm}$. MRI showed the thickened wall of the colon above the anastomosis and the beak sign at the inlet of the pelvis. There were no signs of pelvic recurrence of cancer. Third, most patients could not be cured by endoscopic treatments such as dilation or stent. The majority of patients required a permanent stoma $(66.7 \%)$ or reconstruction of the anastomosis $(22.2 \%)$. Fourth, no acute ischemia or clinically significant anastomotic leakage was detected during primary sphincter-saving surgery. Fifth, radiation-induced fibrosis of the colon above the anastomosis was diagnosed by endoscopic or pathological examination.

The present study suggested that the IMRT was associated with RIPCO. A previous meta-analysis of 859 patients from six studies showed that IMRT was associated with lower incidences of $\geq$ grade 3 acute overall gastrointestinal toxicity, diarrhea, and proctitis compared with 3DCRT [8]. Compared with conventional chemoradiotherapy, IMRT can also significantly reduce the dose distribution to the anal sphincters for patients with rectal cancer [13]. Whether IMRT can decrease the dose distribution to proximal resection margins and radiation injuries need further studies.

A small BMI was associated with RIPCO. There are several potential explanations for the results observed in this study. Optimal radiotherapy delivery is affected by obesity, and the tumor response may be impaired [14]. In the RIPCO group, more patients had a small BMI. It has been reported that autologous fat may play a potential role in the treatment of radiation-induced fibrosis [15]. This study suggested that a BMI less than $21.1 \mathrm{~kg} / \mathrm{m} 2$ was an independent predictor of RIPCO.

Sacral depth more than $4.1 \mathrm{~cm}$ was associated with RIPCO. The stricture colon in a deep sacrum may be easy to form the sacral flexure in an acute angle after primary surgery, with the compression of thickened soft tissues, including bladder, seminal vesicles, muscles, and lateral fascia (Fig. 1E). In addition to radiation injuries of the colon and rectum, adhesions and fibrosis in a narrow pelvis after NCRT and surgery may restrict the dilation space of the colon. Due to radiation-induced fibrosis, the soft tissue may become thicker and compress the neorectum in the pelvis [6]. Hence, a deep sacrum may exacerbate the stenosis and associate with RIPCO.

Most patients with RIPCO were diagnosed after stoma closure, although water-soluble contrast enema was performed before closure. The insufficient display of the colonic lumen may have resulted in the missing diagnosis before stoma closure (Fig. 1G). To decrease the missed diagnosis rate before stoma closure, we recommend that using at least $250 \mathrm{ml}$ of Urografin with sufficient pressure to fill and display the colonic lumen thoroughly during the examination (Fig. 1H). It may help to identify RIPCO and anastomotic leakage before stoma closure. Masaaki also reported a 13\% recurrence of anastomosis leakage even though anastomotic healing was confirmed with water-soluble contrast enema before 
closure. Their study showed that ischemia at the anastomotic site was the main risk factor for recurrent leakage [16]. The PILLAR study suggested that the ICG could change $7.9 \%$ of the surgical plan because of the insufficient blood supply above the anastomosis [17]. However, no acute ischemia was detected from proctoscope during primary surgery in our study, and radiation-induced microvascular stenosis or occlusion and chronic ischemia may have led to RIPCO after NCRT and sphincter-saving surgery.

To date, there have been few effective methods to prevent and reverse radiation-induced intestinal fibrosis implemented in the clinic [19]. Hence, some surgeons recommend the addition of extended proximal colectomy and taking down the splenic flexure with the descending colon for anastomosis during primary sphincter-saving surgery. Previous studies suggested that the radiation injuries and fibrosis of proximal resection margins in patients with RIPCO were more severe than those without RIPCO [20,21]. Therefore, we proposed that a colonic stricture above the anastomosis was "the criminal colon" and should be removed during primary surgery after NCRT. The proposed proximal margin was $19.0(14.8-25) \mathrm{cm}$ in patients with RIPCO in this study. This was consistent with the previous study. It suggested that radiation injury occurred within $20 \mathrm{~cm}$ proximal to rectal cancer [21]. However, whether it is necessary to routinely perform extended colectomy remains controversial.

This study has some limitations. First, this was a retrospective study with a limited number of patients in a single center. There may be selection bias. Second, the records of some important parameters were missing, including the mobilization of the splenic flexure, the length of the proximal bowel, the radiation dose distributions for the proximal margins, the MRI signals, and the radiation injury scores of the proximal margin during primary surgery. Because the sigmoid colon was usually long enough, in this study, we did not mobilize the splenic flexure routinely for all patients and performing an extended colectomy with the descending colon for anastomosis as recommended by experienced surgeons [8, 22]. However, this is one of the very few studies that provided the incidence and the risk assessment, including radiation modalities, for RIPCO. Further studies are needed to assess the radiation injury of the proximal margin and the risk of RIPCO before or during sphincter-saving surgery after NCRT [21]. The results may help with surgical planning and tailored surgery. In addition, to reduce radiation injuries, neoadjuvant therapy with optimal radiation modalities or without radiation in selected patients may decrease the radiation injuries without compromising long-term survival in the future [23,24]. This study will draw attention to prevention for radiation-induced fibrosis.

\section{Conclusion}

RIPCO is a rare but severe complication after sphincter-saving surgery following NCRT. A BMI less than $21.1 \mathrm{~kg} / \mathrm{m}^{2}$, a sacral depth more than $4.1 \mathrm{~cm}$, and the radiation modality were independently associated with RIPCO. IMRT may be associated with decreased incidence of RIPCO. Further studies are needed to validate the results and prevent the complications related to radiation-induced fibrosis.

\section{Abbreviations}


NCRT, neoadjuvant chemoradiotherapy; RIPCO, radiation-induced pelvic colorectal obstruction; SPS, sphincter-saving surgery; IMRT, Intensity-modulated radiotherapy; 3DCRT, three-dimensionally conformal radiation; $\mathrm{BMI}$, body mass index; PTV, planning target volume; PI, the pelvic inlet length; PU, pubic tubercle height; PO, pelvic outlet length; SAL; sacral length; SDE, sacral depth; ISD, interspinous distance.

\section{Declarations}

\section{Funding}

This study was supported by Fujian Minimally Invasive Medical Center Construction Project (No. 2017 171), Joint Funds for the innovation of Science and Technology, Fujian province (No. 2017Y9038), Ethicon Excellence in Surgery Grant of Wu Jieping Medical Foundation (No.320.2710.1845, No.320.2710.1833) and Healthcare Joint fund of Fujian Provincial Natural Science Foundation Projects (No. 2018J01183).

\section{Availability of data and materials}

The datasets used and/or analyzed during the current study are available from the corresponding author on reasonable request.

\section{Ethics approval and consent to participate}

All procedures performed in the present study were in accordance with the ethical standards and written informed consent has been obtained for each patient. The study has been approved by the ethics committee of Fujian Medical University Union Hospital (reference number 2019KY100).

\section{Consent for publication}

Not applicable.

\section{Competing interests}

The authors declare that they have no competing interests.

\section{Authors' contributions}

$\mathrm{SH}, \mathrm{YH}$ and $\mathrm{PC}$ had the idea and took the lead in writing the manuscript. SH, MC, YC carried out the data acquisition. $\mathrm{SH}$ performed the analytic calculations and designed the figures. $\mathrm{SH}, \mathrm{MC}, \mathrm{YC}, \mathrm{XW}$ contributed 
to the interpretation of the results. SH, MC, YC, XW, WJ, XL, YH, PC discussed the results, contributed to the final manuscript. All authors read and approved the final manuscript.

\section{Acknowledgment}

The authors thank Benhua Xu in Department of Radiotherapy at Fujian Medical University Union Hospital.

\section{References}

1. NCCN Clinical Practice Guidelines in Oncology Rectal Cancer. Version 1, 2020. Available at: http://www.nccn.org/professionals/physician_gls/pdf/rectal.pdf.

2. Mignanelli ED, de Campos-Lobato LF, Stocchi L, Lavery IC, Dietz DW. Downstaging after chemoradiotherapy for locally advanced rectal cancer: is there more (tumor) than meets the eye? Dis Colon Rectum. 2010;53:251-6.

3. Battersby NJ, Bouliotis G, Emmertsen KJ, et al. Development and external validation of a nomogram and online tool to predict bowel dysfunction following restorative rectal cancer resection: the POLARS score. Gut. 2018;67(4):688-96.

4. Qin $\mathrm{Q}, \mathrm{Ma} T$, Deng $\mathrm{Y}$, et al. Impact of preoperative radiotherapy on anastomotic leakage and stenosis after rectal cancer resection: post hoc analysis of a randomized controlled trial. Dis Colon Rectum. 2016;59(10):934-42. doi:10.1097/DCR.0000000000000665.

5. Paquette IM, Vogel JD, Abbas MA, et al. The American society of colon and rectal surgeons clinical practice guidelines forthe treatment of chronic radiation proctitis. Dis Colon Rectum. 2018;61(10):1135-40.

6. Chi P, Chen Z, Gao Y, et al. Diagnosis and treatment of pelvic wall and bowel fibrosis with bowel obstruction induced by neoadjuvant chemoradiotherapy for rectal carcinoma[J]. Zhonghua Wei Chang Wai Ke Za Zhi; 2015, 18(11):1092-1097.

7. Huang SH, Chi P, Lin HM, et al. Rectovaginal fistula for middle and low rectal cancer in the era of neoadjuvant chemoradiotherapy and total mesorectal excision. European Surgery-Acta Chirurgica Austriaca 2015; 47: SUPPL. 1 (S68).

8. Hiranyakas A, Silva GD, Denoya P, et al. Colorectal anastomotic stricture: Is it associated with inadequate colonic mobilization? Tech Coloproctol. 2013;17(4):371-5.

9. Wee $\mathrm{CW}$, Kang $\mathrm{HC}, \mathrm{Wu} \mathrm{HG}$, et al. Intensity-modulated radiotherapy versus three-dimensional conformal radiotherapy in rectal cancer treated with neoadjuvant concurrent chemoradiation: metaanalysis and pooled-analysis of acute toxicity. Jpn J Clin Oncol. 2018;48(5):458-66.

10. Yamaoka Y, Yamaguchi T, Kinugasa Y,et al. Mesorectal fat area as a useful predictor of the difficulty of robotic-assisted laparoscopic total mesorectal excision for rectal cancer. Surg Endosc. 2019;33(2):557-66. 
11. Huang $S$, Chen $M$, Deng $Y$, et al. Mesorectal fat area and mesorectal area affect the surgical difficulty of robotic-assisted mesorectal excision and intersphincteric resection respectively in different ways. Colorectal Dis. 2020 Feb 10. doi: 10.1111/codi.15012. [Epub ahead of print].

12. Lee SY, Kim CH, Kim YJ, et al. Anastomotic stricture after ultralow anterior resection or intersphincteric resection for very low-lying rectal cancer[J]. Surg Endosc. 2018;32(2):660-6.

13. Dapper H, Rodríguez I, Münch S, et al. Impact of VMAT-IMRT compared to 3D conformal radiotherapy on anal sphincter dose distribution in neoadjuvant chemoradiation of rectal cancer. Radiat Oncol. 2018;13(1):237.

14. Sun $Y, X u Z$, Lin $H$, et al. Impact of body mass index on treatment outcome of neoadjuvant chemoradiotherapy in locally advanced rectal cancer. Eur J Surg Oncol. 2017;43(10):1828-34.

15. Kumar R, Griffin M, Adigbli G, et al. Lipotransfer for radiation-induced skin fibrosis. Br J Surg. 2016;103(8):950-61.

16. Kitaguchi D, Nishizawa Y, Sasaki T, et al. Recurrence of rectal anastomotic leakage following stoma closure: assessment of risk factors. Colorectal Dis. 2019;21(11):1304-11.

17. Jafari MD, Wexner SD, Martz JE, et al. Perfusion assessment in laparoscopic left-sided/anterior resection (PILLAR II): a multi-institutional study. J Am Coll Surg. 2015;220:82-92.

18. Hauer-Jensen M, Denham JW, Andreyev HJ. Radiation enteropathy-pathogenesis, treatment and prevention. Nature reviews gastroenterology hepatology. 2014;11(8):470-9.

19. Wu P, Zhong Q, Ma T, et al. To what extent should the intestinal be resected proximally after radiotherapy: hint from a pathological view. Gastroenterology Report. 2019 oct; goz047, https://doi.org/10.1093/gastro/goz047 available on: https://academic.oup.com/gastro/advancearticle-pdf/doi/10.1093/gastro/goz047/30302271/goz047.pdf.

20. Chen Z. Experimental Study of Optical Diagnosis with Multiphoton Imaging and Intervention with Pirfenidone for Radiation-induced Pelvic Fibrosis [D]. Fuzhou: Fujian Medical University Union Hospital; 2016. [in Chinese].

21. Qin Q, Zhu Y, Wu P, et al. Radiation-induced injury on surgical margins: a clue to anastomotic leakage after rectal-cancer resection with neoadjuvant chemoradiotherapy? Gastroenterology Report. Gastroenterol Rep (Oxf). 2019;7(2):98-106.

22. Qin Q, Kuang Y, Ma T, et al. Efficacy analysis of proximally extended resection for locally advanced rectal cancer after neoadjuvant chemoradiotherapy [J]. Zhonghua Wei Chang Wai Ke Za Zhi. 2017;20(11):1256-62.

23. Deng Y, Chi P, Lan P, et al. Neoadjuvant Modified FOLFOX6 With or Without Radiation Versus Fluorouracil Plus Radiation for Locally Advanced Rectal Cancer: Final Results of the Chinese FOWARC Trial.J Clin Oncol. 2019; 37(34):3223-3233.

24. Glynne-Jones R, Wyrwicz L, Tiret E, et al. ESMO Rectal cancer: ESMO Clinical Practice Guidelines for diagnosis, treatment and follow-up. Ann Oncol. 2018;29(Suppl 4):iv263.

\section{Figures}




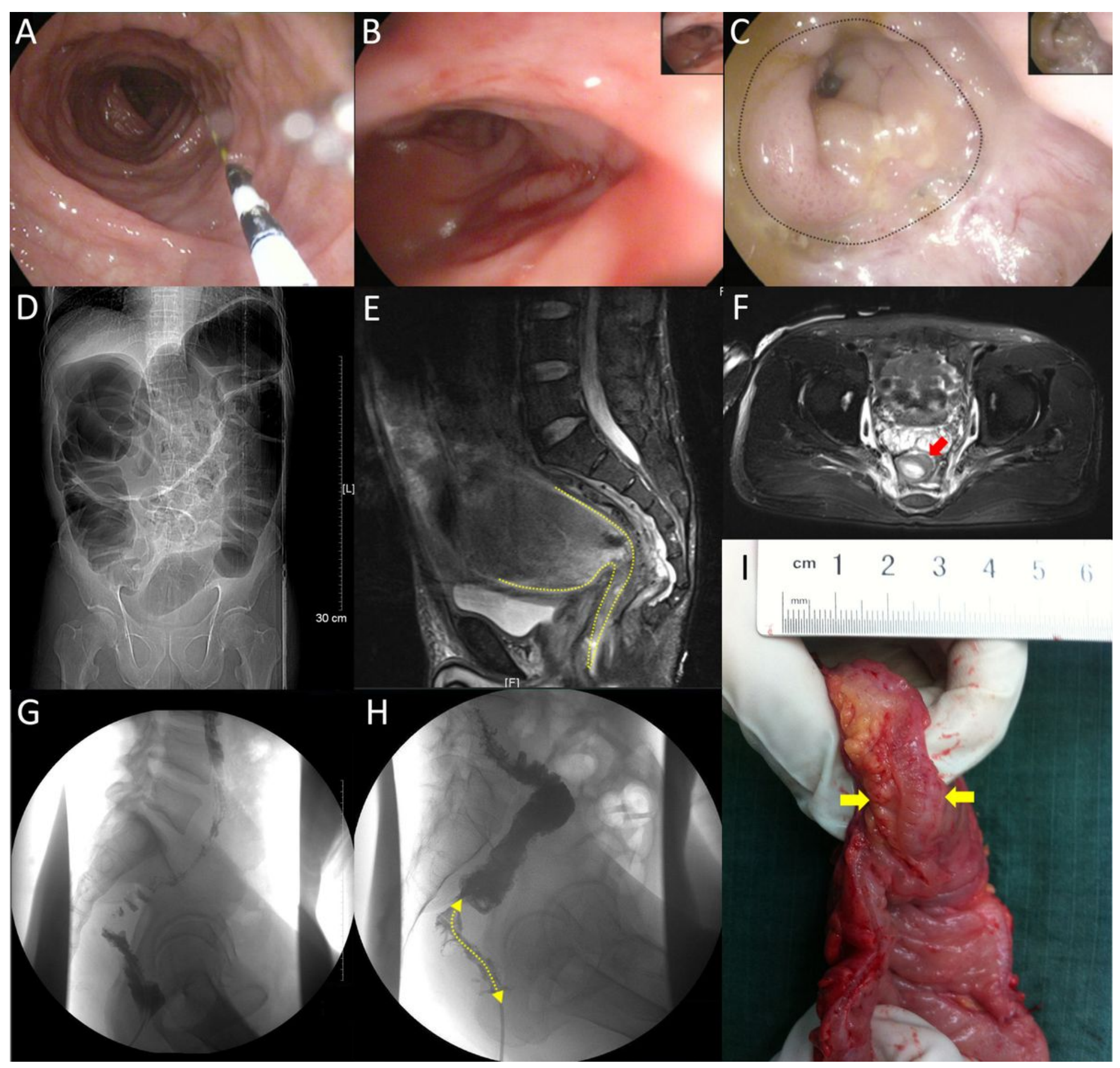

\section{Figure 1}

The endoscopic and radiologic characteristics of RIPCO The descending colon was normal (1A), while the colon in the pelvis was swelling and stricture with the mucosa fold decline (1B). The diameter of the anastomosis was wide enough (the black dotted line in 1C), but there was obvious stenosis in the proximal colon. The manifestation of RIPCO was a megacolon (1D), beak sign with dilated colon above the pelvis and stricture in the pelvis (the yellow dotted line in 2E), and the thickened bowel wall (the red arrow in 2F). The water-soluble contrast enema with an insufficient filling of the colon could not detect the stenosis and missed the diagnosis before stoma closure $(1 \mathrm{G})$. By contrast, after stoma closure, it displayed the colonic stricture (the double-arrow dotted line in $1 \mathrm{H}$ ). The specimen after a redo surgery 
showed the thickened bowel wall (between the two yellow arrows, 1I) with a diagnosis of radiationinduced intestinal fibrosis in the pathological examination.

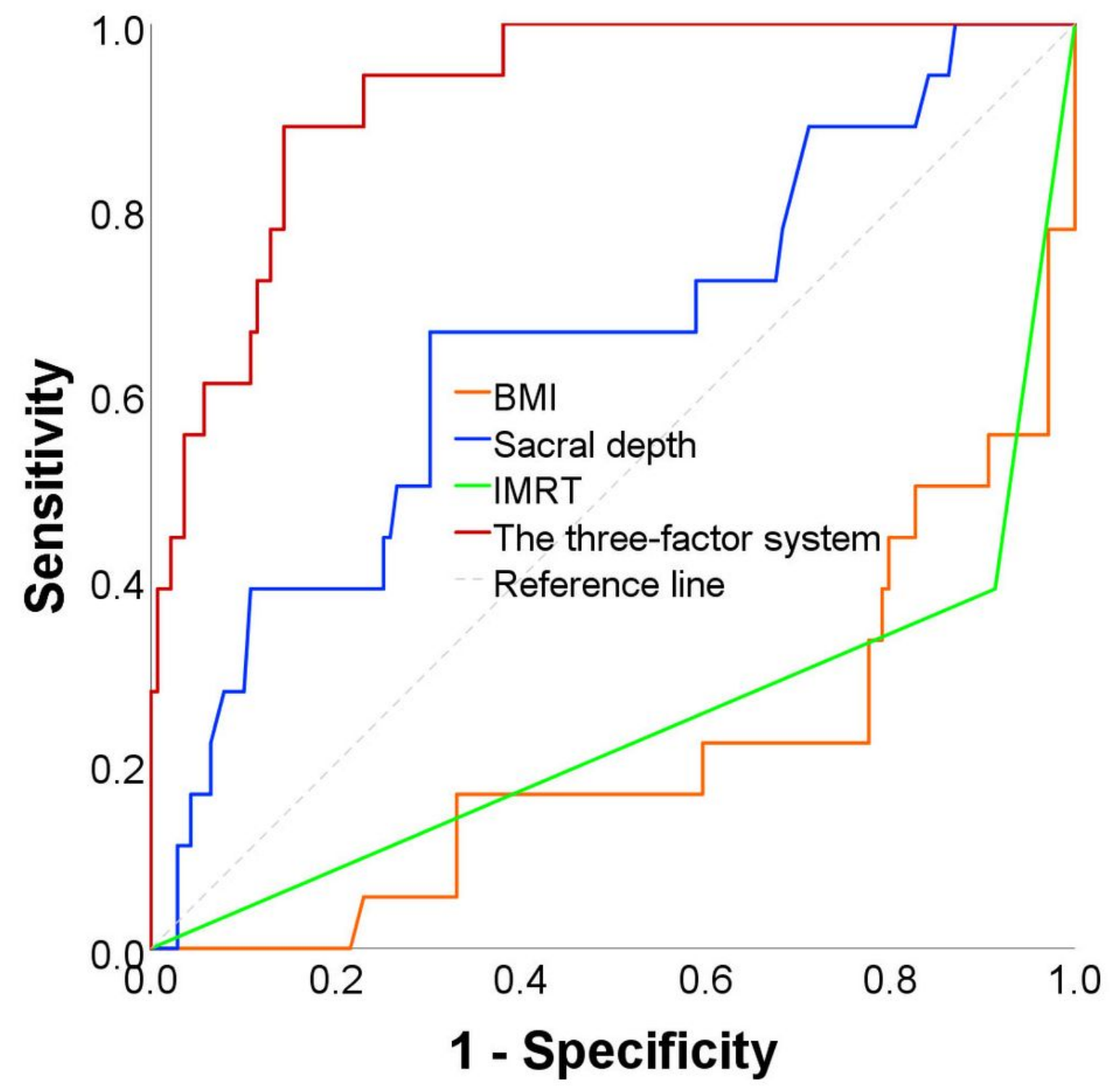

Figure 2

The ROC curves of independent parameters associated with RIPCO The ROC curves showed that the cutoff values of BMI and sacral depth were $21.1 \mathrm{~kg} / \mathrm{m} 2$ and $4.1 \mathrm{~cm}$, respectively. The area under the curve of the three-factor model was 0.921 . With a cutoff value of 0.121 , the sensitivity was $88.9 \%$, and the specificity was $85.6 \%$. 


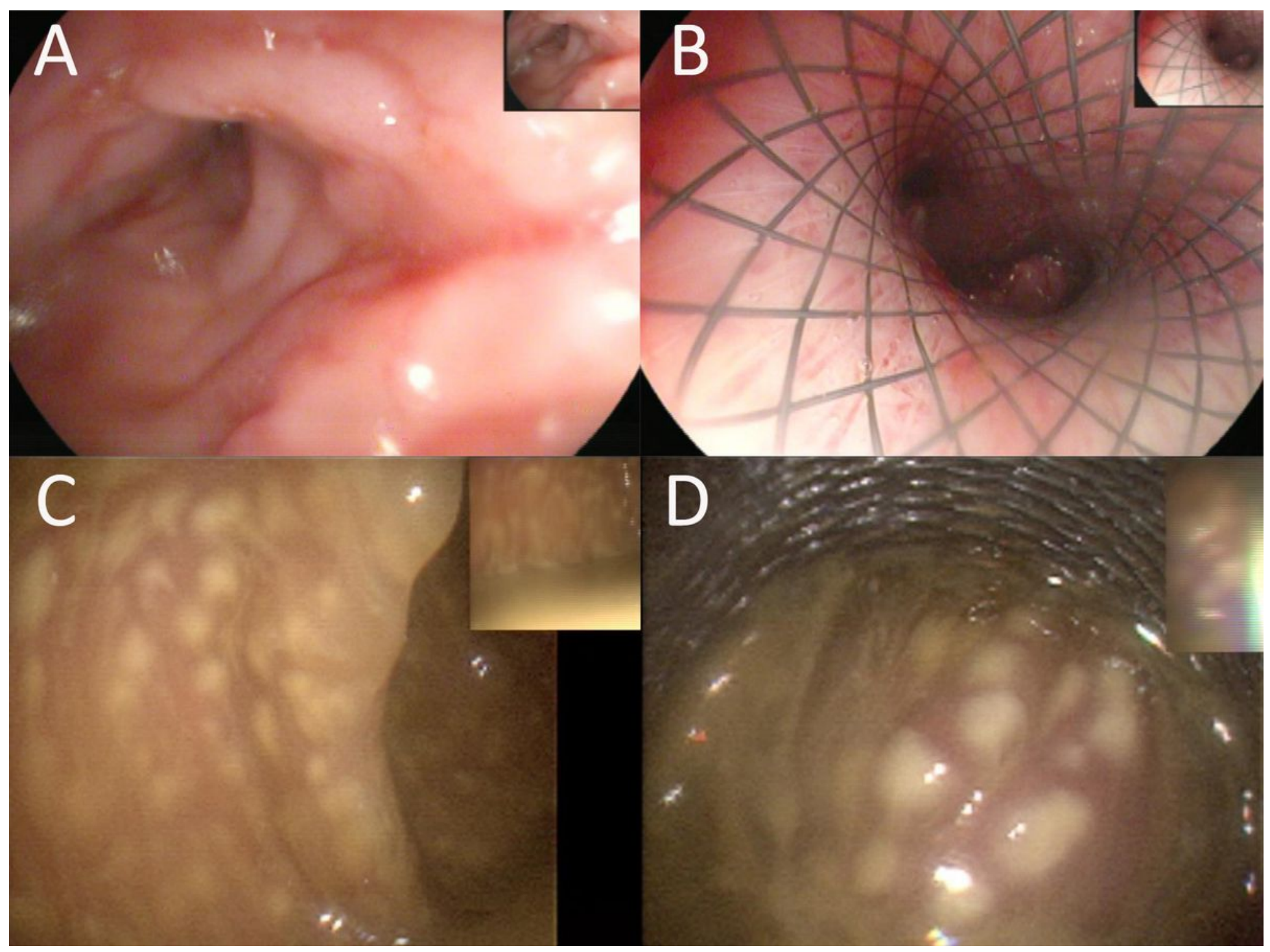

\section{Figure 3}

A 37-year-old male with RIPCO developed the Clostridium difficile infection after endoscopic stent placement. A 37-year-old male received neoadjuvant chemoradiotherapy and ultra-low anterior resection developed RIPCO after the first stoma closure. After the placement of an endoscopic stent, he developed the Clostridium difficile infection. The endoscopic image before (3A) and after stent placement (3B) showed that stenosis was dilated unfavorably. The patient developed a Clostridium difficile infection 5 days later (3C and $3 D$ ). We removed the stent, inserted a transanal tube, and cured the infection with Vancomycin. One month later, the patient underwent a redo surgery with intersphincteric resection and ileostomy. After the second stoma closure, the Wexner score was 5. 